\title{
Intraocular pressure changes and corneal biomechanics after hyperopic small-incision lenticule extraction
}

Dan Fu ${ }^{1,2,3+}$, Meiyan $\mathrm{Li}^{1,2,3+}$, Michael C. Knorz ${ }^{4}$, Shengsheng Wei ${ }^{5}$, Jianmin Shang ${ }^{1,2,3}$ and Xingtao Zhou ${ }^{1,2,3^{*}}$

\begin{abstract}
Background: We aimed to compare the intraocular pressure (IOP) measurements by a dynamic Scheimpflug analyzer (Corvis ST), a non-contact tonometer, and an ocular response analyzer after hyperopic small-incision lenticule extraction (SMILE).

Methods: Thirteen patients who underwent hyperopic SMILE in one eye were enrolled prospectively. IOP and corneal biomechanical parameters were measured preoperatively and at 1 week, 1 month, and 3 months postoperatively with a non-contact tonometer $\left(\mathrm{IOP}_{\mathrm{NCT}}\right)$, Corvis ST (biomechanical corrected IOP [bIOP]), and ocular response analyzer (Goldmann-correlated intraocular pressure [IOPg] and cornea compensated IOP [IOPcc]). A linear mixed model was used to compare the IOPs and biomechanical values among methods at each time point.

Results: $1 O P_{\mathrm{NCT}}, \mathrm{IOPg}$, and IOPCC dropped significantly after surgery, with the amplitude being $3.15 \pm 0.48 \mathrm{mmHg}$, $5.49 \pm 0.94 \mathrm{mmHg}$, and $4.34 \pm 0.97 \mathrm{mmHg}$, respectively, at the last follow-up visit. IOP ${ }_{\mathrm{NCT}}$ decreased by $0.11 \pm 0.06$ $\mathrm{mmHg}$ per $\mu \mathrm{m}$ of excised central corneal thickness. blOP did not change significantly after surgery. Preoperatively, no difference was found among the four measurements $(P>0.05)$. Postoperatively, IOP $\mathrm{NCT}_{\text {and }}$ blOP were higher than IOPg and IOPcc. blOP was independent of cornea thickness at last follow-up visit, whereas it correlated significantly with corneal biomechanics similar to the other three IOP values.
\end{abstract}

Conclusion: bIOP is a relative accurate measure of IOP after hyperopic SMILE.

Keywords: Hyperopia, Small-incision lenticule extraction, Intraocular pressure, Corneal biomechanics

\section{Background}

Cornea refractive surgery corrects the refractive error by removing a part of the corneal tissue, and consequently changes both the corneal shape and corneal biomechanics. Previous studies have identified this procedure to change the intraocular pressure (IOP) measurements [1, 2].

\footnotetext{
* Correspondence: doctzhouxingtao@163.com

${ }^{\dagger}$ Dan Fu and Meiyan Li contributed equally to this work and should be assigned as equal first authors

'Department of Ophthalmology, Eye and ENT Hospital of Fudan University, No. 83 FenYang Road, Shanghai 200031, China

${ }^{2} \mathrm{NHC}$ Key Laboratory of Myopia (Fudan University), Shanghai, China

Full list of author information is available at the end of the article
}

Among the nearly 10 million refractive procedures performed, it is estimated that more than 200,000 eyes may be at risk of a missed glaucoma diagnosis based on a conservative $2 \%$ incidence of glaucoma [3]. It is generally accepted that IOP measurements falsely decrease after corneal myopic refractive surgery. This phenomenon is observed after photorefractive keratectomy (PRK), laser in situ keratomileusis (LASIK), and myopic small incision lenticule extraction (SMILE) [1, 4].

The principle of hyperopic correction, which differs from that of myopic correction, is to make the central cornea steeper. This is achieved with SMILE by creating a concave lenticule that is thinnest in the central area. 
Liu [5] reported that hyperopic SMILE can cause more distortion of collagen fibril formation than myopic SMILE in animal models, and therefore, changes in IOP measurements after hyperopic SMILE may be different from that measured after myopic SMILE. Schallhorn et al. [4] reported that hyperopic ablations (both PRK and LASIK) cause lower IOP measurements, smaller in magnitude than that calculated after myopic ablations, and that this decrease in IOP was weakly correlated with preoperative spherical equation after hyperopic LASIK but not hyperopic PRK. Because of the absence of flap in SMILE and the use of a different laser, it would be of great interest to explore the IOP changes induced by hyperopic SMILE.

The current study aimed to explore the effect of hyperopic SMILE on IOP assessment using different measurement methods.

\section{Methods}

\section{Subjects}

Thirteen patients with hyperopic (13 eyes) were enrolled prospectively between March 2017 and June 2018 at the Eye and ENT Hospital of Fudan University (Table 1). Approval was obtained from the institutional ethics committee, and all patients signed informed consent. All procedures adhered to the tenets of the Declaration of Helsinki.

Inclusion criteria were as follows: age $\geq 18$ years; sphere +2 to +6.0 diopters (D), with astigmatism up to $3.0 \mathrm{D}$; the difference between manifest and cycloplegic refraction was no more than $1.0 \mathrm{D}$.

Patients with abnormal topography, dilated pupil size less than $7 \mathrm{~mm}$, history of intraocular surgery, and glaucoma were excluded.

Preoperative examinations included slit lamp examination, objective and subjective refraction assessments, uncorrected distance visual acuity (UDVA) measurement, corrected distance visual acuity (CDVA) measurement, corneal tomography with a rotating Scheimpflug camera (Pentacam, Oculus, Wetzlar, Germany), and fundus examination.

Table 1 Baseline information of enrolled patients

\begin{tabular}{lll}
\hline & Hyperopia group & (range) \\
\hline Age $(\mathrm{y})$ & $32.8 \pm 9.0$ & $(18-45)$ \\
Male $(\%)$ & $3 / 13$ & $/$ \\
Spherical diopter $(\mathrm{D})$ & $4.17 \pm 1.55$ & $(2.00-6.00)$ \\
Cylinder $(\mathrm{D})$ & $-0.90 \pm 0.75$ & $(-2.25-0.00)$ \\
$\mathrm{CCT}(\mu \mathrm{m})$ & $546.7 \pm 25.3$ & $(507.0-601.0)$ \\
Km $(\mathrm{D})$ & $42.26 \pm 1.12$ & $(40.60-44.70)$ \\
Lenticule thickness $(\mu \mathrm{m})$ & $89.0 \pm 24.0$ & $(46.0-132.0)$ \\
\hline
\end{tabular}

CCT central corneal thickness, $\mathrm{Km}$ mean keratometry
IOP and corneal biomechanics measurement

IOP $_{\text {NCT }}$ (non-contact IOP): The non-contact tonometer (TX-20, Canon, Tokyo, Japan) was used. One average value was automatically calculated from 3 measurements. Reproducibility of measurements was identified previously [6].

\section{bIOP (biomechanical corrected IOP)}

The Corvis ST (Corneal Visualization Scheimpflug Technology instrument; Oculus, Wetzlar, Germany) is a Scheimpflug-based dynamic corneal tonometer, which incorporates the corneal biomechanics and IOP parameters. The system uses an algorithm to calculate biomechanically corrected IOP (bIOP) and compensates for changes in corneal thickness and stiffness [7].

\section{IOPg (Goldmann-correlated IOP), IOCcc (cornea compensated IOP), CRF (corneal resistance factor), and $\mathrm{CH}$ (corneal hysteresis)}

These four values were derived from ocular response analyzer (ORA; Reichert Ophthalmic Instruments, Depew, NY, USA). ORA uses an air puff to deform the cornea. Because of its viscoelastic nature, the cornea resists the air puff, resulting in different values for the inward and outward flexing, which is termed as corneal hysteresis $(\mathrm{CH})$. The corneal resistance factor (CRF) represents the resistance of cornea [8]. Each eye was measured 4 times, and only measurements with a waveform score greater than 5 were used for further analysis.

Two corneal biomechanical parameters derived from Corvis ST were analyzed. A1 Time (first applanation time), and HC DA (deformation amplitude, the largest anterior- posterior displacement of the cornea apex at the highest concavity phase) were the most repeatable and reproducible parameters [9]. SP-A1 (resultant pressure [adjusted pressure at A1 (adj AP1) - biomechanically compensated IOP (Biop)] divided by deflection amplitude at A1) was a new parameter which was used to describe the cornea stiffness, higher values of which meant a more stiff cornea [2].

All measurements were performed by the same examiner (FD) to decrease inter-observer variability and were taken at approximately the same time of day.

\section{Surgical techniques}

All surgeries were performed by the same surgeon (ZXT). After standard sterile draping, all patients were treated with the VisuMax laser (Carl Zeiss Meditec AG, Jena, Germany, version 3.1) with repetition rate of 500 $\mathrm{kHz}$ and pulse energy of $30 \mathrm{~nJ}$. The following settings were used for hyperopic SMILE: the cap diameter was $8.8 \mathrm{~mm}$ and the thickness $120 \mu \mathrm{m}$; the optical zones ranged between $5.3-6.3 \mathrm{~mm}$, with a $2-\mathrm{mm}$ transition 


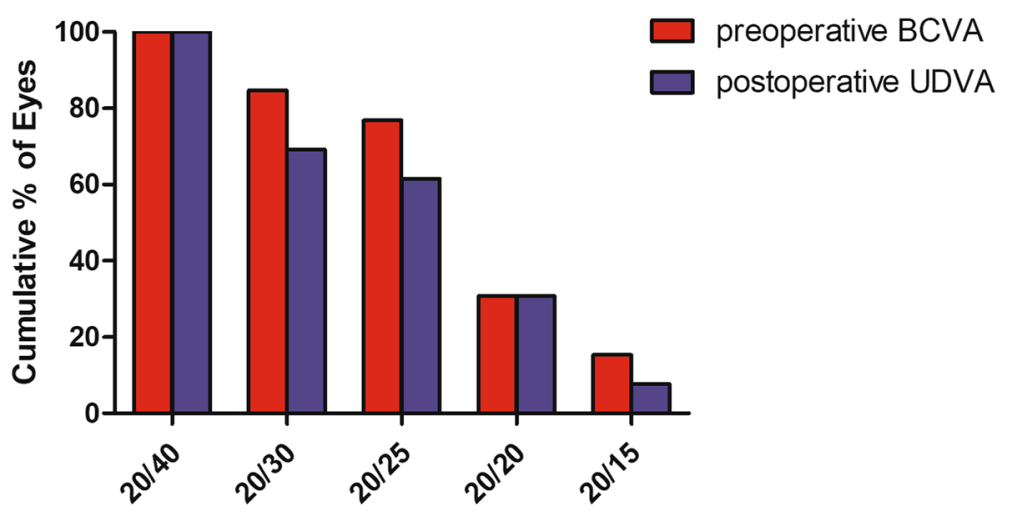

Fig. 1 Preoperative corrected visual acuity and postoperative visual acuity at the last visit

zone; a single $2.0-\mathrm{mm}$ side cut was made at the 12 o'clock position with an angle of $90^{\circ}$.

The detailed steps of SMILE have been described previously [10]. Total suction time was approximately $35 \mathrm{~s}$. After lenticule scanning, the surgeon used a splitter to separate the upper interface, following the lower lenticule interface separation. The lenticule was then removed by superior incision. Thereafter, the surgeon examined the cornea with a built-in slit lamp to detect whether parts of the lenticule remained. One drop of prednisolone and levofloxacin was instilled at the end of the surgery.

All surgeries were performed successfully, with no intraoperative or postoperative complications.

Postoperatively, the patients were instructed to use fluorometholone eye drops 8 times a day, and to reduce the usage frequency by 1 every 3 days (totally 24 days). Artificial tears were prescribed for 3 to 4 weeks, for use as needed.

\section{Follow-up}

Patients were examined at 1 week, 1 month, and 3 months postoperatively. At each follow-up visit, the visual acuity, subjective refraction, corneal topography, and IOP measurements were performed using three devices.

\section{Statistical analyses}

All data were recorded and analyzed using SPSS (version 22, IBM Corp, USA). First, the Kolmogorov-Smirnov test was used to check the normality of data. Linear mixedmodel analysis of variance with post hoc least significant difference multiple comparisons were used to compare the postoperative IOP measurements between different visits and different methods at the same visit. The Spearman rank correlation was used to assess the corneal biomechanical parameters obtained from the Corvis ST and to determine potential postoperative factors affecting the postoperative IOP measurements. $P<0.05$ was considered statistically significant.

\section{Results}

All patients completed the 3-months follow-up visit. The safety index (postoperative CDVA/preoperative CDVA) was $0.96 \pm 0.12$, and the efficacy index (postoperative UDVA/preoperative CDVA) was $0.93 \pm 0.14$ at the last visit. (Fig. 1) The refraction at each visit time is shown in Table 2.

\section{Changes in IOP measurement}

The IOP values at different points of time are shown in Table 3. Preoperatively, no difference was found among the measurements. At 1 week postoperatively, the $\mathrm{IOP}_{\mathrm{NCT}}$ was $2.52 \pm 1.11 \mathrm{mmHg}$ higher than $\mathrm{IOPg}(P=$ $0.04)$; bIOP was $2.32 \pm 0.85 \mathrm{mmHg}$ higher than IOPg $(P=0.02) ;$ IOP $_{\mathrm{NCT}}$ and bIOP values showed no difference. At 1 month postoperatively, bIOP was $3.60 \pm 0.89$ $\mathrm{mmHg}$ higher than IOPg $(P=0.004)$ and $3.32 \pm 0.86$ higher than IOPcC $(P=0.005)$, and $\operatorname{IOP}_{\mathrm{NCT}}$ was $2.56 \pm$ $0.50 \mathrm{mmHg}$ higher than IOPg $(P=0.001)$. No difference was found between $\mathrm{IOP}_{\mathrm{NCT}}$ and bIOP values. At 3 months postoperatively, bIOP was the highest IOP value (IOP ${ }_{\mathrm{CC}}: \Delta=3.29 \pm 0.63 \mathrm{mmHg}, P=0.001 ;$ IOPg: $\Delta=$ $3.68 \pm 0.91 \mathrm{mmHg}, \quad P=0.003 ; \quad \mathrm{IOP}_{\mathrm{NCT}}: \Delta=2.13 \pm 0.70$ $\mathrm{mmHg}, P=0.01)$. IOPg and IOPcc values showed no difference at all postoperative visits.

Except bIOP, compared with the preoperative values, the other three measurements were lower postoperatively. $\mathrm{IOP}_{\mathrm{NCT}}$ remained stable from before surgery to 1 month after the surgery (postoperative 1 month vs postoperative 3 months, $\Delta=1.85 \pm 0.82 \mathrm{mmHg}, P=0.04)$, and decreased $3.15 \pm 0.48 \mathrm{mmHg}$ at postoperative 3 months compared with the preoperative values $(P<0.001$;

Table 2 Mean refraction(D) at each visit time

\begin{tabular}{lllll}
\hline & Pre-op & Post-1w & Post-1 m & Post-3 m \\
\hline Spherical diopter & $4.2 \pm 1.5$ & $-0.1 \pm 1.4$ & $0.2 \pm 1.5$ & $0.5 \pm 1.0$ \\
cylinder & $-0.9 \pm 0.7$ & $-0.5 \pm 0.5$ & $-0.5 \pm 0.5$ & $-0.5 \pm 0.4$
\end{tabular}

$D$ diopter 
Table 3 Mean IOP values $(\mathrm{mmHg})$ of the four methods at each time point

\begin{tabular}{llllll}
\hline & pre & Post-1w & Post-1 m & Post-3 ms & $P$ \\
\hline $\mathrm{IOP}_{\mathrm{NCT}}$ & $15.40 \pm 3.20$ & $14.73 \pm 3.74$ & $13.87 \pm 3.96$ & $12.08 \pm 2.83$ & 0.006 \\
$\mathrm{blOP}$ & $15.77 \pm 4.18$ & $14.21 \pm 1.87$ & $14.30 \pm 1.76$ & $14.13 \pm 1.61$ & 0.878 \\
$\mathrm{IOPg}$ & $15.23 \pm 4.84$ & $11.75 \pm 3.56$ & $10.36 \pm 3.19$ & $10.51 \pm 3.83$ & $<0.001$ \\
$\mathrm{IOP} C \mathrm{CC}$ & $14.62 \pm 3.94$ & $11.54 \pm 1.07$ & $10.46 \pm 2.83$ & $10.90 \pm 2.80$ & $<0.001$ \\
$\mathrm{P}$ & 0.928 & 0.045 & $<0.001$ & $<0.001$ & \\
\hline
\end{tabular}

$I O P_{N C T}$ non-contact intraocular pressure, biop biomechanical corrected intraocular pressure, $1 \mathrm{OPg}$ Goldmann-correlated intraocular pressure, $1 \mathrm{PCC}$ cornea compensated intraocular pressure

$0.11 \pm 0.06 \mathrm{mmHg}$ reduction per micro removed cornea tissue $\left[\Delta \mathrm{IOP}_{\mathrm{NCT}} /\right.$ lenticule thickness]).

Compared with the preoperative values, IOPcc started to decrease at postoperative 1 week $(\Delta=2.71 \pm 1.04$ $\mathrm{mmHg}, P=0.03)$, and decreased until 1 month postoperatively $(\Delta=4.94 \pm 1.25 \mathrm{mmHg}, P=0.006)$. IOPg decreased by $4.30 \pm 1.13 \mathrm{mmHg}(P=0.007)$ at 1 week postoperatively and remained stable thereafter. Among all 4 measurements at postoperative 3 months postoperatively, IOPg showed the greatest difference between pre- and postoperative values $(\Delta=5.49 \pm 0.94 \mathrm{mmHg}, P=0.001)$ (Fig. 2$)$.

\section{Changes in corneal biomechanical and correlation analysis}

The biomechanical parameters from ORA and Corvis ST are shown in Table 4. CRF and SP-A1 dropped significantly after surgery $(P<0.05)$. $\mathrm{CH}, \mathrm{A} 1$ Time and HC DA showed no significant difference among the follow-up visits.

Using Spearman analysis, at postoperative 3 months follow-up, HC DA was negatively related with all IOPs ( $\mathrm{r}$ ranges from -0.82 to $-0.74, P<0.05)$. IOPg and IOPcc were correlated with CRF $(r=0.68-0.91 ; P<0.05)$ and postoperative CCT $(r=0.83-0.95 ; P<0.01)$. bIOP was independent of preoperative CCT as well as postoperative CCT and correlated with A1 Time $(r=0.87 ; P=0.001)$ and HC DA $(r=-0.74 ; P=0.01)$ at the last postoperative follow-up visit. $\mathrm{IOP}_{\mathrm{NCT}}$ at the last postoperative follow-up visit correlated with homologous CRF, A1 Time, and HC DA as well as preoperative $\mathrm{IOP}_{\mathrm{NCT}}(r=0.86 ; P=0.001)$ (Table 5 ).

\section{Discussion}

Accurate IOP measurement is extremely important for ophthalmologists because false low readings of IOP may delay the diagnosis of ocular hypertension or glaucoma [11]. In this study, we evaluated the effect of hyperopic SMILE on different IOP measurement techniques. To our best knowledge, it is the first report of this kind.

In this study, the average decrease of IOP measurements from pre- to postoperatively ranged from $0.42-5.48 \mathrm{mmHg}$ among the different measurement techniques used. Lee [12] reported that 6 months postoperatively, $\mathrm{IOP}_{\mathrm{NCT}}$ decreased by $2.04 \pm 1.44 \mathrm{mmHg}$ after myopic transepithelial PRK and by $2.63 \pm 1.60 \mathrm{mmHg}$ after myopic femtosecond-LASIK. Li [13] demonstrated that $\triangle \mathrm{IOP}_{\mathrm{NCT}}$ per micrometer of ablated tissue after 6 months postoperatively was $0.05 \pm 0.02 \mathrm{mmHg}$ in myopic SMILE group, and $0.05 \pm 0.03 \mathrm{mmHg}$ in myopic femtosecond-LASIK group, which was lesser than $0.11 \pm$ $0.06 \mathrm{mmHg}$ calculated in the present study. Reinstein et al. [14] found that postoperative tensile strength was greatest after SMILE, followed by PRK, and was lowest after LASIK. Thus, different corneal stiffness impairments may partially account for the different IOP reduction among surgeries. In addition, epithelium preservation and flap-free procedures may result in difference in pressure resistance. Moreover, the hyperopic lenticule, different from myopic ones, is thinnest at the center and causes less thinning of the central cornea. It may lead to different wound healing processes, although the direct relationship between would healing and IOP measurement remains to be identified [15].

The present study used four IOP measurement methods; three of them attempted to correct for the biomechanical changes of the cornea caused by corneal refractive surgery. We found that bIOP (biomechanical corrected IOP, measured with the Corvis ST) most

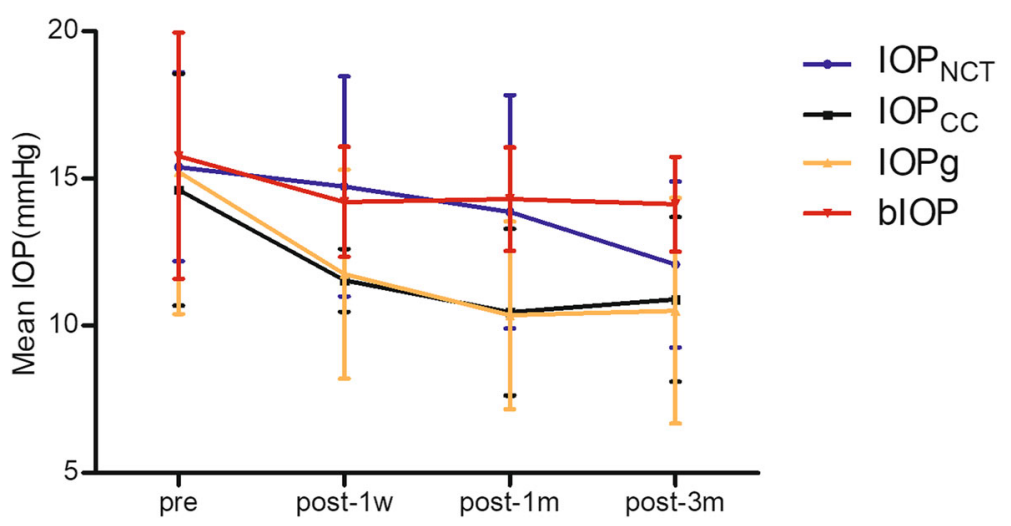

Fig. 2 Changes in mean intraocular pressure measurement during postoperative follow-up 
Table 4 Corneal biomechanical parameters measured using the ocular response analyzer and Corvis ST

\begin{tabular}{llllll}
\hline & Pre-op & Post-1w & Post-1 m & Post-3 ms & P \\
\hline CH & $11.47 \pm 1.47$ & $10.88 \pm 1.19$ & $11.05 \pm 1.95$ & 0.13 \\
CRF & $11.25 \pm 2.22$ & $9.71 \pm 1.64$ & $10.15 \pm 2.33$ & $9.51 \pm 1.87$ & .001 \\
A1 Time (ms) & $7.10 \pm 0.52$ & $6.87 \pm 0.24$ & $6.93 \pm 0.57$ & $6.88 \pm 0.31$ & 0.23 \\
HC DA (mm) & $1.03 \pm 0.14$ & $1.13 \pm 0.14$ & $1.14 \pm 0.15$ & $1.11 \pm 0.08$ & 0.12 \\
SP -A1 & $114.87 \pm 16.67$ & $93.64 \pm 18.00$ & $92.08 \pm 20.82$ & $94.03 \pm 19.07$ & 0.001 \\
\hline
\end{tabular}

$\mathrm{CH}$ cornea hysterisis, CRF cornea resistance factor, A1 Time first applanation time, HC DA deformation amplitude, the largest anterior-posterior displacement of the corneal apex at the highest concavity phase, SP-A1 Resultant pressure [adjusted pressure at A1 (adj AP1) - biomechanically compensated IOP (Biop)] divided by deflection amplitude at $\mathrm{A} 1$

closely matched the approximate preoperative IOP values, whereas the other three estimated IOP values were lower after hyperopic SMILE. Similar results were observed after myopic LASIK and myopic SMILE [16, 17]. Lee [12] also reported that bIOP values remained unchanged after myopic LASIK and PRK. Previous studies have demonstrated that CCT can influence IOP measurement. Liu et al. [18] reported that IOP readings may have a $2.87-\mathrm{mmHg}$ range because of CCT variations, but have a much larger $17.26-\mathrm{mmHg}$ range because of changes in corneal biomechanical properties alone. Biomechanical properties therefore seem to have a much greater effect on IOP than CCT. In this study, we found that both cornea biomechanics and CCT correlated with the IOP values. However, we found no correlation between pre and postoperative CCT values for bIOP; they were similar after both hyperopic and myopic SMILE, indicating it is a reliable assessment method postoperatively.

In this study, IOPg and IOPcc remained constant at all postoperative follow-up visits, and both values correlated with CCT as well as cornea biomechanics. This was

Table 5 Correlations between IOPs and corneal biomechanical parameters at 3 months visit ( $r$ [P])

\begin{tabular}{|c|c|c|c|c|}
\hline & $\mathrm{IOP}_{\mathrm{NCT}}$ & blOP & $\mathrm{IOPg}$ & IOPCC \\
\hline$\overline{\mathrm{CH}}$ & $0.48(0.16)$ & $0.28(0.44)$ & $0.56(0.09)$ & $0.22(0.54)$ \\
\hline CRF & $0.75(0.01)$ & $0.59(0.07)$ & $0.91(0.001)$ & $0.68(0.03)$ \\
\hline $\begin{array}{l}\text { A1 Time } \\
\text { (ms) }\end{array}$ & $0.66(0.03)$ & $\begin{array}{l}0.87 \\
(0.001)\end{array}$ & $0.71(0.03)$ & $0.64(0.06)$ \\
\hline $\begin{array}{l}\text { HC DA } \\
(\mathrm{mm})\end{array}$ & $\begin{array}{l}-0.76 \\
(0.006)\end{array}$ & $\begin{array}{l}-0.74 \\
(0.01)\end{array}$ & $\begin{array}{l}-0.82 \\
(0.007)\end{array}$ & $\begin{array}{l}-0.76 \\
(0.02)\end{array}$ \\
\hline SP A1 & $0.49(0.15)$ & $0.32(0.37)$ & $0.72(0.04)$ & $0.49(0.22)$ \\
\hline $\mathrm{CCT}(\mu \mathrm{m})$ & $0.49(0.11)$ & $0.32(0.31)$ & $0.95(0.001)$ & $\begin{array}{l}0.83 \\
(0.003)\end{array}$ \\
\hline Km (D) & $-0.05(0.89)$ & $0.29(0.36)$ & $-0.24(0.51)$ & $-0.36(0.30)$ \\
\hline
\end{tabular}

$r$ correlation coefficient, CCT central cornea thickness at 3 month after surgery, $\mathrm{CH}$ cornea hysterisis, $\mathrm{CRF}$ cornea resistance factor, A1 Time first applanation time, HC DA deformation amplitude, the largest anterior-posterior displacement of the corneal apex at the highest concavity phase, SP-A1 Resultant pressure [adjusted pressure at A1 (adj AP1) - biomechanically compensated IOP (Biop)] divided by deflection amplitude at A1, IOPNCT noncontact intraocular pressure, biop biomechanical corrected intraocular pressure, $1 \mathrm{OPg}$ Goldmann-correlated intraocular pressure, IOPcc cornea compensated intraocular pressure consistent with the results of Mollan, [19], but contrary to that reported by Sullivan-Mee [20], who reported that IOPcc was higher than IOPg. Different types of ocular pathologies may be responsible for this discrepancy. Sullivan-Mee [20] measured IOPg and IOPcc in patients with suspected or diagnosed glaucoma, whereas Mollan examined patients with keratoconus and a control group. IOPg, an average value of inward and outward pressures, is considered identical with that observed with Goldmann applanation tonometry [21]. IOPcc is affected to a less degree by variations in corneal thickness and corneal biomechanical properties. The present results indicate that IOPg and IOPcC show no difference for hyperopic SMILE related IOP changes.

$\mathrm{IOP}_{\mathrm{NCT}}$ was the most commonly used clinical parameter. In this study, $\mathrm{IOP}_{\mathrm{NCT}}$ decreased by $3.15 \pm 0.48$ $\mathrm{mmHg}$ after hyperopic SMILE, greater than bIOP, but less than IOPg and IOPcc. Although Wolfs et al. reported a positive correlation of IOP with CCT, [22] we found no significant correlation between preoperative CCT and IOP $_{\mathrm{NCT}}$ values in this study. In addition, $\mathrm{IOP}_{\mathrm{NCT}}$ at last follow-up visit correlated with preoperative $\mathrm{IOP}_{\mathrm{NCT}}$ and some corneal biomechanical properties, suggesting that corneal biomechanics but not CCT may be of greater importance when predicting $\mathrm{IOP}_{\mathrm{NCT}}$ after hyperopic SMILE. Additional studies are warranted to further validate these findings.

This study has several limitations. The study lacks corresponding measurements with Goldmann applanation tonometer, which is considered the gold standard reference for IOP measurements. The enrolled cases had astigmatism, which did not lead to pure hyperopia correction, though cornea thickness and biomechanics are major factors affecting IOP and mixed astigmatism might probably have some effect on the results. In addition, the sample size in the current study is small, and a larger sample size and longer study duration are needed.

\section{Conclusion}

In conclusion, $\mathrm{IOP}_{\mathrm{NCT}}$ decreased after hyperopic SMILE, and its value correlates with preoperative $\mathrm{IOP}_{\mathrm{NCT}}$ as well as corneal biomechanical properties. bIOP seems to be an accurate parameter to assess postoperative IOP. 


\section{Abbreviations}

IOP: Intraocular pressure; SMILE: Small-incision lenticule extraction; IOP ${ }_{\text {NCT: Non-contact tonometer; bIOP: Biomechanical corrected IOP; }}$ ORA: Ocular response analyzer; IOPg: Goldmann-correlated intraocular pressure; IOPcc: Cornea compensated IOP; LASIK: Laser in situ keratomileusis; PRK: Photorefractive keratectomy; UDVA: Uncorrected distance visual acuity; CDVA: Corrected distance visual acuity; CH: Corneal hysteresis; CRF: Cornea resistant factor; A1 Time: First applanation time; HC DA: Deformation amplitude, the largest anterior- posterior displacement of the cornea apex at the highest concavity phase; SP- A1: Resultant pressure [adjusted pressure at A1 (adj AP1) - biomechanically compensated IOP (Biop)] divided by deflection amplitude at $\mathrm{A} 1$

\section{Acknowledgements}

Not applicable.

\section{Authors' contributions}

LMY and WSS analyzed and interpreted the patient data. SJM and FD performed the clinical ocular examinations, and were major contributors in writing the manuscript. KM and ZXT contributed to major revise and study design. All authors read and approved the final manuscript.

\section{Funding}

National Natural Science Foundation of China for Young Scholars (Grant No. 81500753)

The receiver is $L M Y$, one of the first author in present study, contributing to interpreting the patient data.

Natural Science Foundation of China (Grant No. 81570879) \& (Grant No. 81770955),

Joint Research Project of New Frontier Technology in Municipal Hospitals (SHDC12018103) Project of Shanghai Science and Technology (Grant No .17140902900) (Grant No. 17411950200).

Three fundings above are received by ZXT, who is corresponding author in this study, contributing to study design and major correction of whole study.

\section{Availability of data and materials}

The datasets used and analyzed during the current study are available from the corresponding author on reasonable request.

\section{Ethics approval and consent to participate}

Approval was obtained from the ethics committee of Eye and ENT Hospital of Fudan University, and informed consent was signed by each patient.

\section{Consent for publication}

Not applicable.

\section{Competing interests}

The authors declare that they have no competing interests.

\section{Author details}

'Department of Ophthalmology, Eye and ENT Hospital of Fudan University, No. 83 FenYang Road, Shanghai 200031, China. ${ }^{2}$ NHC Key Laboratory of Myopia (Fudan University), Shanghai, China. ${ }^{3}$ Shanghai Research Center of Ophthalmology and Optometry, Shanghai, China. ${ }^{4}$ Medical Faculty Mannheim of the University of Heidelberg, Mannheim, Germany. ${ }^{5}$ Xi'An Forth Hospital, Shanxi, China.

Received: 21 November 2019 Accepted: 11 March 2020

Published online: 05 April 2020

\section{References}

1. Hosny M, Aboalazayem F, El Shiwy H, Salem M. Comparison of different intraocular pressure measurement techniques in normal eyes and post small incision lenticule extraction. Clin Ophthalmol. 2017;11:1309-14.

2. Fernandez J, Rodriguez-Vallejo M, Martinez J, Tauste A, Salvestrini P, Pinero DP. New parameters for evaluating corneal biomechanics and intraocular pressure after small-incision lenticule extraction by Scheimpflug-based dynamic tonometry. J Cataract Refract Surg. 2017:43(6):803-11.

3. Samuelson TW. Refractive surgery in glaucoma. Curr Opin Ophthalmol. 2004;15(2):112-8.
4. Schallhorn JM, Schallhorn SC, Ou Y. Factors that influence intraocular pressure changes after myopic and hyperopic LASIK and photorefractive keratectomy: a large population study. Ophthalmology. 2015;122(3):471-9.

5. Liu YC, Ang HP, Teo EP, Lwin NC, Yam GH, Mehta JS. Wound healing profiles of hyperopic-small incision lenticule extraction (SMILE). Sci Rep. 2016;6:29802.

6. Yilmaz I, Altan C, Aygit ED, Alagoz C, Baz O, Ahmet S, Urvasizoglu S, Yasa D, Demirok A. Comparison of three methods of tonometry in normal subjects: Goldmann applanation tonometer, non-contact airpuff tonometer, and Tono-pen XL. Clin Ophthalmol. 2014;8:1069-74.

7. Joda AA, Shervin MM, Kook D, Elsheikh A. Development and validation of a correction equation for Corvis tonometry. Comput Methods Biomech Biomed Engin. 2016;19(9):943-53.

8. Matsuura M, Hirasawa K, Murata H, Nakakura S, Kiuchi Y, Asaoka R. The usefulness of CorvisST tonometry and the ocular response analyzer to assess the progression of glaucoma. Sci Rep. 2017;7:40798.

9. Hon Y, Lam AK. Corneal deformation measurement using Scheimpflug noncontact tonometry. Optom Vis Sci. 2013;90(1):e1-8.

10. Li M, Zhao J, Miao H, Shen Y, Sun L, Tian M, Wadium E, Zhou X. Mild decentration measured by a Scheimpflug camera and its impact on visual quality following SMILE in the early learning curve. Invest Ophthalmol Vis Sci. 2014:55(6):3886-92.

11. Shrivastava A, Madu A, Schultz J. Refractive surgery and the glaucoma patient. Curr Opin Ophthalmol. 2011;22(4):215-21.

12. Lee H, Roberts CJ, Kim TI, Ambrosio R Jr, Elsheikh A, Yong Kang DS. Changes in biomechanically corrected intraocular pressure and dynamic corneal response parameters before and after transepithelial photorefractive keratectomy and femtosecond laser-assisted laser in situ keratomileusis. J Cataract Refract Surg. 2017;43(12):1495-503.

13. Li H, Wang Y, Dou R, Wei P, Zhang J, Zhao W, Li L. Intraocular pressure changes and relationship with corneal biomechanics after SMILE and FSLASIK. Invest Ophthalmol Vis Sci. 2016;57(10):4180-6.

14. Reinstein DZ, Archer TJ, Randleman JB. Mathematical model to compare the relative tensile strength of the cornea after PRK, LASIK, and small incision lenticule extraction. J Refract Surg. 2013;29(7):454-60.

15. Dupps WJ Jr, Wilson SE. Biomechanics and wound healing in the cornea. Exp Eye Res. 2006;83(4):709-20

16. Hong JX, Yu ZQ, Jiang CH, Zhou XT, Liu ZG, Sun XH, Xu JJ. Corvis ST tonometer for measuring postoperative IOP in LASIK patients. Optom Vis Sci. 2015;92(5):589-95.

17. Chen KJ, Joda A, Vinciguerra R, Eliasy A, Sefat SMM, Kook D, Geraghty B, Roberts CJ, Elsheikh A. Clinical evaluation of a new correction algorithm for dynamic Scheimpflug analyzer tonometry before and after laser in situ keratomileusis and small-incision lenticule extraction. J Cataract Refract Surg. 2018;44(5):581-8.

18. Liu J, Roberts CJ. Influence of corneal biomechanical properties on intraocular pressure measurement: quantitative analysis. J Cataract Refract Surg. 2005;31(1):146-55.

19. Mollan SP, Wolffsohn JS, Nessim M, Laiquzzaman M, Sivakumar S, Hartley S, Shah S. Accuracy of Goldmann, ocular response analyser, Pascal and TonoPen $\mathrm{XL}$ tonometry in keratoconic and normal eyes. Br J Ophthalmol. 2008;92(12):1661-5.

20. Sullivan-Mee M, Gerhardt G, Halverson KD, Qualls C. Repeatability and reproducibility for intraocular pressure measurement by dynamic contour, ocular response analyzer, and goldmann applanation tonometry. J Glaucoma. 2009;18(9):666-73.

21. Fabian ID, Barequet IS, Skaat A, Rechtman E, Goldenfeld M, Roberts CJ, Melamed S. Intraocular pressure measurements and biomechanical properties of the cornea in eyes after penetrating keratoplasty. Am J Ophthalmol. 2011;151(5):774-81.

22. Wolfs RC, Klaver CC, Vingerling JR, Grobbee DE, Hofman A, de Jong PT. Distribution of central corneal thickness and its association with intraocular pressure: the Rotterdam study. Am J Ophthalmol. 1997;123(6):767-72.

\section{Publisher's Note}

Springer Nature remains neutral with regard to jurisdictional claims in published maps and institutional affiliations. 